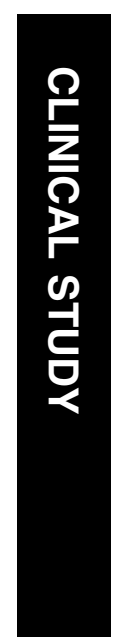

\section{Improvement of horizontal macular contraction after surgical removal of epiretinal membranes}

HK Yang ${ }^{1}$, SJ Kim ${ }^{1}$, YS Jung ${ }^{2}$, KG Kim², JH Kim² and $\mathrm{HG} \mathrm{Yu}^{1,3}$
${ }^{1}$ Department of

Ophthalmology, Seoul National University College of Medicine, Seoul, Korea

${ }^{2}$ Department of Radiology and the Institute of Radiation Medicine, Clinical Research Institute, Seoul National University College of Medicine, Seoul, Korea

${ }^{3}$ Sensory Organs Research Institute, Seoul National University Medical Research Center, Seoul, Korea

Correspondence: HG Yu, Department of

Ophthalmology, Seoul National University Hospital, 103 Daehak-ro, Jongno-gu, Seoul 110-799, Korea

Tel: + 8222072 2438;

Fax: + 8227413187 .

E-mail: hgonyu@snu.ac.kr

Received: 27 May 2010 Accepted in revised form: 16 December 2010 Published online: 1 April 2011

\begin{abstract}
Purpose To determine whether horizontal macular contraction caused by epiretinal membranes (ERMs) improves after surgical removal.

Methods In this prospective, single-center, observational study, 63 consecutive patients with unilateral idiopathic ERM in one eye and no retinal disease in the fellow eye underwent pars plana vitrectomy. Fundus photography and optical coherence tomography (OCT) were performed preoperatively and at 3 months postoperatively. The area enclosed by superior and inferior major vessels from the optic disc to the fovea (area under major vessel (AUV)) and the macroscopic diverging angle (MDA) between superior and inferior major vessels were calculated using digital image analysis of fundus photographs and compared pre- and postoperatively.

Results AUV was significantly smaller in the eyes with ERM compared with the normal fellow eyes $(P<0.001)$. Significant postoperative change in AUV and MDA was demonstrated after ERM removal $(P<0.001)$. However, postoperative AUV of grade 2 and 3 ERM eyes was still significantly smaller than that of normal fellow eyes. Macular thickness differences measured with stratus OCT were positively correlated with AUV differences.

Conclusions Retinal topographic changes caused by ERM improved in part after ERM removal. The improvement of topographic changes were correlated with tomographic changes detected with OCT.

Eye (2011) 25, 754-761; doi:10.1038/eye.2011.48; published online 1 April 2011
\end{abstract}

Keywords: epiretinal membrane; macular contraction; surgical removal
Introduction

Epiretinal membranes (ERMs) range from fine, transparent membranes in the early stages, to thickened and opaque tissues, causing metamorphopsia and severe visual impairment. ${ }^{1}$ As the ERM gets thicker with time, ${ }^{2}$ cellular proliferation and tractional forces at the level of the internal limiting membrane create a puckering effect, inducing the formation of retinal folds radiating outward from the macula. ${ }^{1-4}$ The retinal topographic changes caused by distortion developing in the process of ERM formation are mostly apparent on a simple fundus examination: nerve fiber layer dragging, ectopic fovea, winding corkscrew vessels surrounding the overlying ERM, or major vessel straightening and crowding. ${ }^{1}$

Optical coherence topography (OCT) can quantify cross-sectional morphological changes caused by ERM. ${ }^{5,6}$ The cross-sectional changes can be resolved after surgical removal of ERM and some OCT parameters such as macular thickness are associated with surgical outcome. ${ }^{6}$ However, it is not clear whether retinal topographic changes can be resolved after ERM removal and affect surgical outcome.

To determine whether horizontal macular contraction caused by ERM improves after ERM removal, topographic features related to macular contraction were calculated using digital image analysis of fundus photography and were compared pre- and postoperatively. We also investigated the correlations of topographic features with OCT parameters.

\section{Materials and methods}

Patients and sample collection

This prospective study included consecutive patients who underwent pars plana vitrectomy 
(PPV) with ERM removal for unilateral idiopathic ERM between 1 August 2007 and 31 January 2009 at Seoul National University Hospital. Exclusion criteria included: (1) secondary ERM, such as that caused by diabetic retinopathy, venous occlusion, retinal detachment (RD), uveitis, or trauma; (2) myopia of more than -6 diopters; and (3) other ocular pathologies that had the potential to interfere with functional results, especially severe cataracts of greater than grade 2 nuclear sclerosis and/or cortical opacity; (4) other retinal disease in the fellow eye. ${ }^{7}$

Surgery consisted of standard three-port PPV and ERM peeling using a blunt retinal pick and end-gripping forceps with the use of adjuvant intravitreal steroid during membrane peeling. Preoperative data collection included patient age, gender, operative eye, and history of previous ocular surgery. Complete ophthalmological exams, including best corrected visual acuity (BCVA) using a Snellen line VA chart, refractive error, cataract grading, fundus photography, and stratus OCT, were performed before surgery and at 3 months after surgery. BCVAs were converted into logarithms of minimal angle of resolution (logMAR).

The fellow eye was matched as a control, and the same preoperative and postoperative data mentioned above were collected. The research followed the tenets of the Declaration of Helsinki. Informed consent was obtained from the subjects after explanation of the nature and possible consequences of the study and the research was approved by the institutional review board of the Seoul National University Hospital. We certify that all applicable institutional and governmental regulations concerning the ethical use of human volunteers were followed during this research.

Color fundus photographs of both eyes were taken before surgery and at 3 months after surgery. KOWA VX-10 (Kowa Company Ltd, Tokyo, Japan) and TRC-50IA (Topcon Inc., Tokyo, Japan) fundus cameras were used, and the location of the actual point of fixation was stored while photographs were obtained with target stimulus presentation.

ERM severity was classified into one of three grades on the basis of fundus photography determined through the agreement of two masked observers. This classification of ERM severity has been used in several studies as follows; ${ }^{8,9}$ grade 1 ERM consisted of a transparent membrane causing irregular wrinkling of the inner retina with no ERM edge seen elevating from the retina. Grade 2 ERM was transparent but showed full-thickness macular contraction and membrane edges elevating from the retina along with tortuosity of macular vessels. Grade 3 ERM was opaque, with marked obscuration and distortion of the underlying retina.

Subgroup analysis regarding the clinical severity of ERM was carried out to assess the correlation between quantified measurements of selected topographic features and ERM severity.

Patients were divided into three groups according to the degree of visual improvement at 3 months postoperatively. The 'better' group contained those with a visual improvement of at least two Snellen lines; the 'same' group contained those with a BCVA increase or decrease of up to one Snellen line; the 'worse' group contained those with a BCVA reduction of more than one Snellen line. BCVAs were converted into logMAR.

\section{Image analysis}

In this study, the quantitative change of macular contraction occurring before and after ERM removal was assessed using a semiautomatic image analysis method. A software for digital image processing was developed; major vessels were identified and segmented with directional filtering and adaptive thresholding techniques; the center of the optic disc and fovea were determined, and the preoperative and postoperative quantitative features were calculated semiautomatically from the registered pair of images.

The process of the image analysis program is as follows. The program first extracts major superior and inferior vessels as reference structures for use in the measurement of macular contraction; one from the superior half of the retina, the other from the inferior half. As there may be multiple major vessel branches in the retina, it arbitrarily chooses two major vessels that satisfy our selection criteria, namely, largest diameter compared with nearby vessels, good contrast, and well traced without discontinuity from the optic disc to the vertical line passing through the fovea. The selected vessels were confirmed by two masked observers and they showed absolute agreement for all photographs. A binary image of extracted major vessels is created by applying a series of image segmentation techniques (Figure 1). Second, the centers of the fovea and the optic disc are determined as anatomic landmarks. The fovea is determined by the mean point of fixation. ${ }^{10}$ The center of the optic disc is determined by edge detection of the inferior and nasal disc margins. The landmarks were confirmed by two masked observers with absolute agreement for all photographs.

To quantify macular contraction, two features were used: the area under major vessels (AUV) from the optic disc to the fovea and the macroscopic diverging angle (MDA) of the superior and inferior major vessels. AUV was defined as the area of pixels (pixel $\times$ pixel) enclosed by the superior and inferior major vessels between two vertical lines; the first line passing through the fovea, the second passing through the center of the 
optic disc (Figure 2, left). MDA was defined as the diverging angle of the superior and inferior major vessels $\left({ }^{\circ}\right.$ ). Two intersection points were determined by the crossing points of two major vessels and the vertical line passing through the fovea. The MDA was the angle between two lines: one passing from the center of the optic disc and superior intersection point, the other passing from the center of the optic disc and inferior intersection point (Figure 2, right). Three repeated measures were executed and the mean value was taken for analysis of each feature. The standard deviation (SD) of repeated measurements was assessed for repeatability and validity of the software.
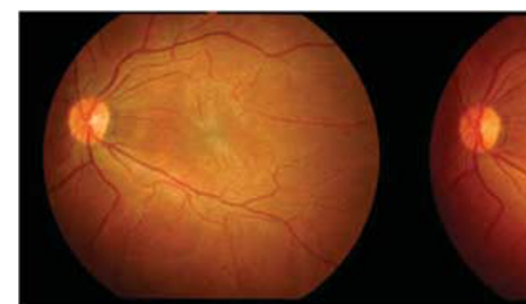

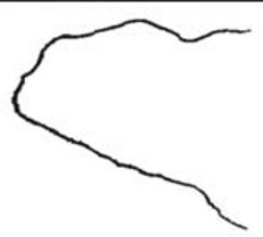

Preoperative

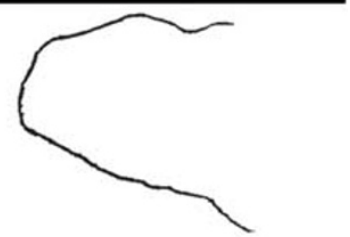

Postoperative
Figure 1 An example of a preoperative and postoperative image pair from a patient with an epiretinal membrane. A patient's fundus photographs are shown (top left) preoperatively with an epiretinal membrane and (top right) postoperatively. The binary images of major vessels from (bottom left) preoperative and (bottom right) postoperative fundus photographs are shown.

\section{Statistical analysis}

Statistical analyses were performed using SPSS for Windows (version 15.0, Statistical Package for the Social Sciences, SPSS Inc., Chicago, IL, USA). The paired $t$-test was used to compare preoperative and postoperative values of retinal topographic features, along with differences in preoperative and postoperative values between ERM and fellow eyes (control). The differences in topographic features occurring before and after surgery were compared between the eyes with ERM and fellow eyes with the ratio paired $t$-test, using the logarithmic value of postoperative/preoperative ratios between eyes. Subgroup analysis was carried out between groups by one-way ANOVA regarding the clinical grading of ERM. Pearson's correlation was performed between pre- and postoperative topographic features and BCVA. A $P$-value of $<0.05$ was considered statistically significant.

\section{Results}

Of the 70 consecutive patients who were enrolled in the study, 7 were excluded for loss before 3-month followup. None of the 63 patients included in the final analysis had postoperative complications of glaucoma or RD during follow-up. The baseline characteristics of the 63 patients are summarized in Table 1 . The mean age was $62.5 \pm 8.4$ years (range, 36-84). There were 19 men and 44 women. Preoperatively, 56 eyes were phakic and 7 eyes were pseudophakic with posterior chamber intraocular lenses. Preoperative mean BCVA was $0.50 \pm 0.29$

(0.10-1.40). Cataract surgery was performed at the time of PPV in $28(44.4 \%)$ eyes.

Mean BCVA improved at 3 months postoperatively, which was $0.19 \pm 0.24$ ( -0.08 to 1.22 ). Subgroup analysis
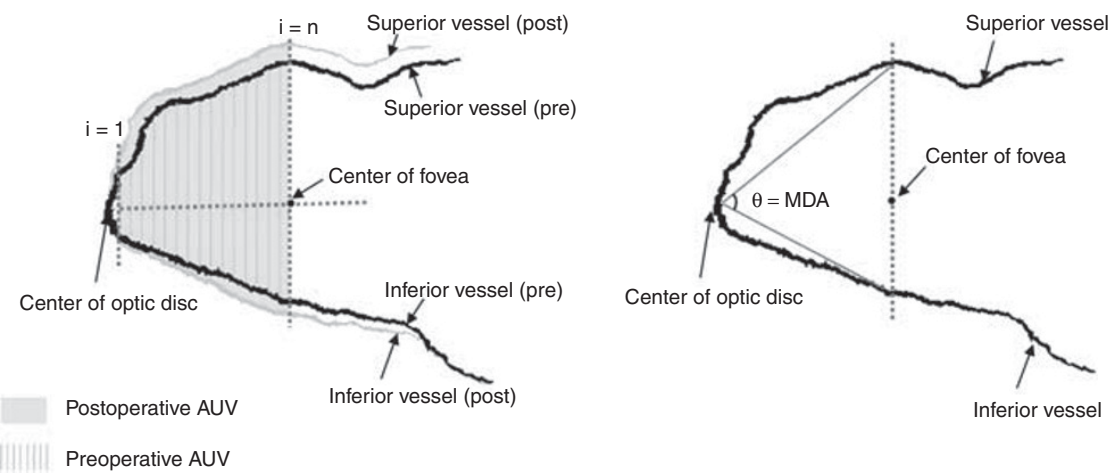

Figure 2 A schematic showing the two features of macular contraction. (Left) Area under major vessels (AUV) in preoperative and postoperative images were calculated from the area enclosed by superior and inferior major vessels from the optic disc to the fovea. (Right) Macroscopic diverging angle (MDA) between superior and inferior major vessels were calculated using digital image analysis of fundus photographs. 
in regard of visual improvement showed that the preoperative ( $P=0.007$ by one-way ANOVA) and postoperative BCVA $(P<0.001)$ were significantly better in the 'better' group compared with other groups.

The preoperative and postoperative values of selected topographic features are summarized in Table 2. AUV was significantly smaller in the eyes with ERM compared to the normal fellow eyes $(P<0.001)$. There was a significant postoperative change in AUV after ERM removal $(P<0.001)$, whereas the fellow eye showed no significant periodic change $(P=0.796)$. According to ERM grading, there was no significant difference in AUV between ERM eyes and fellow eyes pre- and postoperatively in grade 1 ERM (Table 3 ). However, pre- and postoperative AUV of grade 2 and 3 ERM eyes were still significantly smaller than that of normal fellow eyes (Table 3 ). In addition, with the more severe ERM grade, preoperative AUV was smaller with borderline significance $(P=0.073)$, whereas preoperative

Table 1 Preoperative patient characteristics

\begin{tabular}{lc}
\hline Characteristics & Value $(\mathrm{n}=63)$ \\
\hline Mean age at operation (years) & $62.5 \pm 8.4(36-84)$ \\
Male/female gender & $19(30.2 \%) / 44(69.8 \%)$ \\
Laterality & $35 / 28(55.6)$ \\
Right/left (eyes, \%) & $-0.11 \pm 1.43(-5.25$ to +3.75$)$ \\
Refractive error (diopters) & $56(88.9 \%) / 7(10.1)$ \\
Preoperative lens status & $0.50 \pm 0.29(0.10-1.40)$ \\
Phakic/pseudophakic (eyes, \%) & Preoperative BCVA (logMAR)
\end{tabular}

Abbreviations: BCVA, best-corrected visual acuity; logMAR, logarithms of minimal angle of resolution.

All values are given as mean \pm s.d. (range).
CMT was significantly thicker $(P=0.002$ by one-way ANOVA; Table 3).

MDA was smaller in the ERM eyes but it barely reached statistical significance $(P=0.053$; Table 2$)$. There was a significant postoperative change in MDA after ERM removal $(P<0.001)$, whereas the fellow eyes showed no significant periodic change $(P=0.969)$. However, according to EMR grading, MDA showed no significant difference with fellow eyes before or after surgery (Table 3).

The preoperative distance from the optic disc to the fovea was not significantly different between the eyes with ERM and the fellow eyes $(P=0.145)$. No significant postoperative change was noted in the optic disc-tofovea distance after ERM removal $(P=0.376)$.

The repeatability of each features (SD value of three repeated measurements) were 0.33 number of pixels (pixel $\times$ pixel) for AUV, 0.01 degree for MDA.

OCT measures showed that $\triangle C M T$ (postoperative CMT-preoperative CMT) was negatively correlated with $\triangle \mathrm{AUV}$ (postoperative AUV-preoperative AUV; $r=-0.316 ; P=0.014$; Figure 3, left). Preoperative CMT showed positive correlation with $\triangle \mathrm{AUV}(r=0.338$; $P=0.008$ ) and $\triangle \mathrm{MDA}$ (postoperative MDA-preoperative MDA; $r=0.341 ; P=0.008$ ). Postoperative CMT was negatively correlated with preoperative AUV $(r=-0.389 ; P=0.002)$ and postoperative AUV $(r=-0.334 ; P=0.009)$.

Concerning VA parameters, preoperative and postoperative BCVA showed positive correlation with $\triangle \mathrm{AUV}(r=0.327 ; P=0.009, r=0.269 ; P=0.033)$ but $\triangle \mathrm{BCVA}$ (postoperative BCVA-preoperative BCVA) did not show significant correlation $(r=-0.095 ; P=0.458$; Figure 3, middle). With OCT parameters, preoperative

Table 2 Comparison of preoperative and postoperative parameters and postoperative differences between ERM eyes and fellow eyes (control)

\begin{tabular}{|c|c|c|c|c|}
\hline Parameters & $E R M(\mathrm{n}=63)$ & Control $(\mathrm{n}=63)$ & P-value ${ }^{\mathrm{a}}$ & Ratio P-value ${ }^{\mathrm{b}}$ \\
\hline AUV (number of pixels) & & & & $<0.001^{*}$ \\
\hline Preoperative & $16476.1 \pm 3202.1$ & $18181.2 \pm 3121.0$ & $<0.001^{*}$ & \\
\hline Postoperative & $17358.2 \pm 3457.6$ & $18128.8 \pm 3555.9$ & 0.152 & \\
\hline$\Delta \mathrm{AUV}$ & $882.2 \pm 1785.2$ & $-52.4 \pm 1603.3$ & $0.001^{*}$ & \\
\hline$P$-value ${ }^{a}$ & $<0.001^{*}$ & 0.796 & & \\
\hline$M D A$ (degree) & & & & $<0.001^{*}$ \\
\hline Preoperative & $73.4 \pm 11.2$ & $76.5 \pm 8.1$ & 0.053 & \\
\hline Postoperative & $76.1 \pm 10.6$ & $76.5 \pm 7.6$ & 0.781 & \\
\hline$\triangle \mathrm{MDA}$ & $2.7 \pm 2.9$ & $0.0 \pm 2.6$ & $<0.001^{*}$ & \\
\hline$P$-value ${ }^{\mathrm{a}}$ & $<0.001^{*}$ & 0.969 & & \\
\hline
\end{tabular}

Abbreviations: AUV, area under major vessels; ERM, epiretinal membrane; MDA, macroscopic diverging angle.

All values are given as mean \pm s.d.; number of pixels $=$ pixel $\times$ pixel; $\Delta \mathrm{AUV}=$ postoperative $\mathrm{AUV}-$ preoperative $\mathrm{AUV}$; $\Delta \mathrm{MDA}=$ postoperative MDA-preoperative MDA.

a $P$-value by paired $t$-test.

${ }^{\mathrm{b}} P$-value by ratio paired $t$-test of the logarithmic value of (postoperative/preoperative) ratio between both eyes.

${ }^{*} P$-value $<0.05$. 
Table 3 Subgroup analysis of preoperative and postoperative topographic features including central macular thickness according to the clinical grading of epiretinal membrane

\begin{tabular}{|c|c|c|c|c|}
\hline Parameters & Grade $1(\mathrm{n}=19)$ & Grade $2(\mathrm{n}=27)$ & Grade $3(\mathrm{n}=17)$ & $\mathrm{P}-$ value $^{\mathrm{a}}$ \\
\hline \multicolumn{5}{|c|}{ Area under major vessels (number of pixels) } \\
\hline Preoperative & $17843.8 \pm 2264.0$ & $16067.6 \pm 3284.8$ & $15596.3 \pm 3620.1$ & 0.073 \\
\hline Postoperative & $18484.1 \pm 2977.6$ & $16793.3 \pm 3471.5$ & $16997.2 \pm 3819.3$ & 0.235 \\
\hline$P$-value (pre-post) ${ }^{\mathrm{b}}$ & 0.199 & $0.034^{*}$ & $0.002^{*}$ & \\
\hline$P$-value (pre-fellow) ${ }^{c}$ & 0.354 & $0.013^{*}$ & $0.011^{*}$ & \\
\hline$P$-value (post-fellow) ${ }^{\mathrm{c}}$ & 0.948 & $0.031^{*}$ & $0.048^{*}$ & \\
\hline \multicolumn{5}{|c|}{ Macroscopic diverging angle (degree) } \\
\hline Preoperative & $75.7 \pm 8.2$ & $72.1 \pm 12.1$ & $72.8 \pm 12.9$ & 0.552 \\
\hline Postoperative & $77.7 \pm 7.7$ & $75.1 \pm 11.2$ & $75.7 \pm 12.8$ & 0.721 \\
\hline$P$-value (pre-post) ${ }^{\mathrm{b}}$ & $0.002^{*}$ & $<0.001^{*}$ & $0.001^{*}$ & \\
\hline$P$-value $\left(\right.$ pre-fellow) ${ }^{\mathrm{c}}$ & 0.594 & 0.134 & 0.264 & \\
\hline$P$-value (post-fellow) ${ }^{c}$ & 0.921 & 0.763 & 0.968 & \\
\hline \multicolumn{5}{|c|}{ Central macular thickness $(\mu \mathrm{m})$} \\
\hline Preoperative & $344.5 \pm 79.5$ & $395.4 \pm 110.6$ & $468.6 \pm 77.7$ & $0.002^{*}$ \\
\hline Postoperative & $295.1 \pm 48.7$ & $322.5 \pm 62.4$ & $339.9 \pm 80.8$ & 0.122 \\
\hline$P$-value (pre-post) ${ }^{\mathrm{b}}$ & $0.034^{*}$ & $0.001^{*}$ & $<0.001^{*}$ & \\
\hline
\end{tabular}

All values are given as mean \pm s.d.; number of pixels $=$ pixel $\times$ pixel.

${ }^{a} P$-value by one-way ANOVA between different grades of epiretinal membrane.

${ }^{\mathrm{b}} P$-value by paired $t$-test between pre- and postoperative features.

${ }^{\mathrm{c}} P$-value by paired $t$-test between epiretinal membrane eyes and fellow eyes of pre- and postoperative features.

${ }^{*} P$-value $<0.05$.
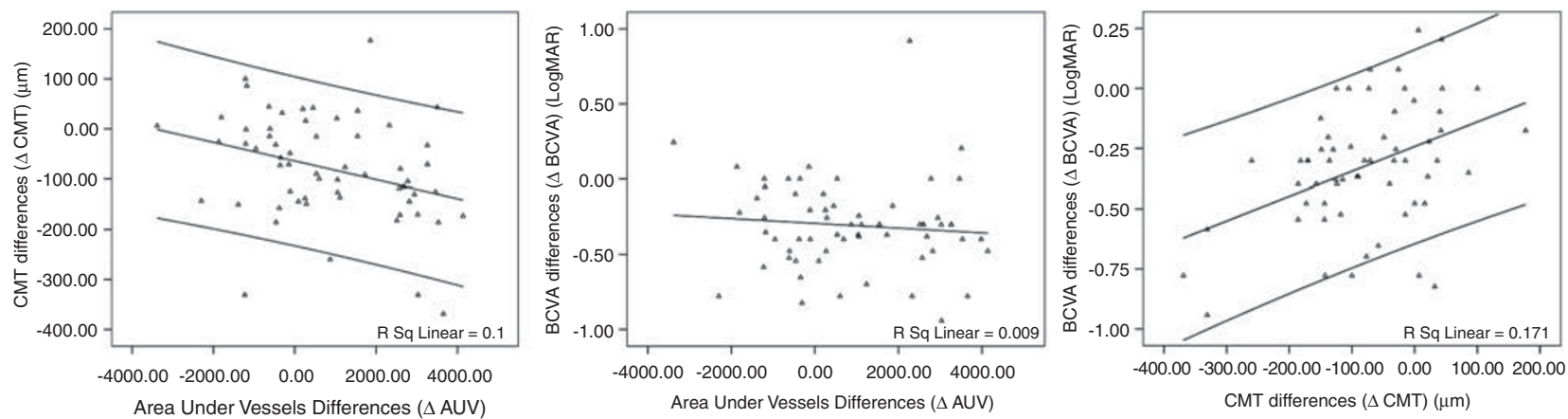

Figure 3 (Left) Linear regression analysis demonstrates negative correlation between area under major vessel differences ( $\triangle$ AUV) and central macular thickness differences $(\triangle C M T)$. (Middle) BCVA differences $(\triangle B C V A)$ did not show significant correlation with $\triangle$ AUV. (Right) $\triangle B C V A$ showed positive correlation with $\triangle \mathrm{CMT}$.

and postoperative BCVA showed positive correlation with preoperative CMT $(r=0.552 ; P<0.001$ for preoperative BCVA, $r=0.341 ; P=0.008$ for postoperative BCVA) and negative correlation with $\triangle \mathrm{CMT}(r=-0.580$; $P<0.001$ for preoperative BCVA, $r=-0.269 ; P=0.038$ for postoperative BCVA). $\triangle \mathrm{BCVA}$ showed negative correlation with preoperative CMT $(r=-0.335, P=0.009)$ and positive correlation with $\triangle \mathrm{CMT}(r=0.413 ; P=0.001$; Figure 3 , right). All three BCVA parameters featured no significant correlation with postoperative CMT $(|r|<0.130, P>0.05)$. Finally, multivariate logistic regression to detect factors affecting visual outcome showed that preoperative BCVA yielded the highest correlation coefficient with postoperative BCVA and $\triangle$ BCVA $(P<0.001)$

\section{Discussion}

The main objective of this study was to quantify changes and improvement in horizontal macular topography followed by surgical removal of ERM, using digital image analysis tools. The results of this study show that along with the localized or regional retinal changes caused by ERM, an overall macular contraction, although not evident by gross appearance, also develops and that contraction is improved after surgical removal. 
The postoperative increases in AUV and MDA after ERM removal may be because of the relief of tangential macular contraction after surgery. ${ }^{11}$ Compared with a previous study that quantified the amount of macular contraction by change in vectors of retinal vessels, which only has use in comparing pre- and post-surgical status, our approach may be useful in detecting the amount of contraction by the absolute value of AUV, especially when compared with the normal fellow eye. ${ }^{5}$ Also, our method is simple, repeatable, and easier to apply.

In this study, unilateral ERM patients were recruited in order to have the fellow eye serve as a control, as the intra-individual differences in fundus features between right and left eyes in healthy subjects may be small. ${ }^{12}$ The preoperative optic disc-to-fovea distance in this study was not significantly different between fellow eyes, indicating that there was little change in the optic discto-fovea distance by retinal contraction because of ERM.

By contrast, preoperative AUV was significantly smaller in ERM eyes compared with fellow eyes. This may be explained by tractional forces associated with ERM development and progression. ${ }^{13}$ Contraction of the membrane exerts tangential traction on the inner retina and overlying vessels, making retinal vessels crowd toward the contraction center. ${ }^{10}$ Thus, a smaller preoperative AUV may indicate more macular contraction caused by ERM. Moreover, AUV showed a decreasing tendency in association with more severe clinical grades of ERM. Therefore, AUV may be regarded as a feature that quantitatively represents horizontal macular contraction and indicates disease progression. $\triangle \mathrm{AUV}$ also showed good correlation with preoperative CMT and $\triangle \mathrm{CMT}$. This indicates that horizontal macular contraction is correlated with vertical retinal contraction in the eyes with ERM.

In addition, although AUV significantly increased after ERM removal, AUV of grade 2 and 3 ERM eyes were still significantly smaller than that of normal fellow eyes. This may be associated with persistent metamorphopsia after successful ERM removal evidenced by OCT in some patients. Also, this finding implies that early surgical removal of ERM, before occurrence of significant topographic change, may be beneficial.

The $\triangle \mathrm{MDA}$ also showed significant change after ERM removal. However, the absolute preoperative and postoperative MDA values were not significantly correlated with gross macular contraction, ERM clinical grades, or vertical distortion measured by CMT. Thus, MDA may be less useful in the quantification of macular contraction compared with AUV and may not be a sensitive indicator of disease progression or recovery.

The relationship between anatomical restoration and functional recovery after surgical ERM removal has been controversial in previous studies. ${ }^{6,14-17}$ In our study,
$\Delta \mathrm{CMT}$ was related to all three visual acuity parameters that are consistent with former studies. ${ }^{6}$ Among horizontal topographic features, only $\triangle \mathrm{AUV}$ was related to preoperative and postoperative visual acuity, yet $\triangle \mathrm{AUV}$ did not show correlation with the amount of $\triangle B C V A$. In addition, multivariate logistic regression revealed that preoperative BCVA yielded the highest correlation with postoperative BCVA and $\triangle \mathrm{BCVA}$. This finding suggests that functional recovery primarily depends on the preoperative status and early intervention. ${ }^{18}$ In recent studies, prolonged macular traction is known to cause irreversible photoreceptor cell loss and disruption of alignment. ${ }^{17,19-21}$ Outer retinal structures rarely return to normal once they are impaired, thus indicating a poor visual prognosis. ${ }^{14}$ Therefore, prompt surgical intervention would be beneficial to prevent such damage. ${ }^{17}$

A confounding factor in the evaluation of functional recovery is that BCVA may underestimate the functional benefit of surgery. ${ }^{22}$ Although subjective visual improvement is achieved in more than $70 \%$ of patients after ERM removal, improvement of metamorphopsia is not measurable by visual acuity. ${ }^{1,11,23,24}$ Other reports have shown a higher number of patients improving in macular sensitivity measured by microperimetry compared with visual acuity testing. ${ }^{22}$ No functional improvement measures other than visual acuity (such as improvement in metamorphopsia or retinal sensitivity) were included in our study, and further studies are warranted. Furthermore, anatomical restoration usually precedes functional recovery, and visual acuity increases have been reported years after surgery. Longer follow-up may, therefore, have resulted in better functional outcomes associated with anatomical improvement. ${ }^{11}$ However, the follow-up period of 3 months may be acceptable, as most patients are known to develop restored maximal functional improvement by 1 or 2 months after surgery. ${ }^{1}$

Another confounding factor is the effect of combined cataract surgery. In former reports, postoperative visual acuity improvement was clearly greater in patients who underwent combined cataract surgery. ${ }^{25}$ However, eyes with severe cataracts were excluded from our study, and there was no significant difference in visual improvement between eyes that underwent concurrent cataract surgery and eyes that did not. A relatively short postoperative follow-up period may have also contributed to this fact.

There are several inherent limitations in interpreting the features measured from fundus photographs. First, direct comparison of the figures should be carried out with caution because of inter-individual differences. The high SD values in MDA and AUV represents this variability (Tables 2 and 3). To correct these errors, the 
postoperative changes in features were also compared in postoperative/preoperative ratio (Table 2). Second, differences in refractive errors may cause discrepancies in the image scales of the photographs. However, refractive errors in our patients ranged from $-5.25 \mathrm{D}$ to $+3.75 \mathrm{D}$, without including any subjects with high myopia or high hyperopia. Anisometropia of $>1.5 \mathrm{D}$ was also rare $(4.8 \%)$, so refractive error-induced image scale discrepancy may not be an important consideration.

In conclusion, macular contraction caused by ERM, which was quantified using digital image analysis, improved after ERM removal. However, with severe ERM grades, horizontal macular contraction only partially resolved to a much smaller value compared to the fellow eye. The strongest prognostic factor related with visual acuity was better preoperative visual acuity, and considering the fact that ERM gets thicker with time, $^{2}$ early surgical intervention in low-grade ERMs might be more beneficial than waiting for progression. Well-designed controlled studies with long term followup would help answer to this clinical decision. Along with OCT, digital image analysis for horizontal macular contraction may serve as a useful guide in clinical practice in various tractional diseases including ERM.

\section{Summary}

\section{What was known before}

- Optical coherence topography (OCT) can quantify cross-sectional morphological changes caused by epiretinal membrane (ERM), which can be resolved after surgical removal of ERM. OCT parameters, such as macular thickness, are associated with surgical outcome.

- However, it is not clear whether macular contraction can be resolved after ERM removal and affect surgical outcome.

What this study adds

- This study quantifies macular contraction using digital image analysis of fundus photography and determines whether horizontal macular contraction caused by ERM improves after srugical removal.

- Macular topographic changes caused by ERM improved in part after ERM removal.

- The improvement of topographic changes were correlated with tomographic changes detected with OCT.

\section{Conflict of interest}

The authors declare no conflict of interest.

\section{Acknowledgements}

This study has been supported in part by grants of the Korea Healthcare technology R\&D Project, Ministry for
Health, Welfare \& Family Affairs, Republic of Korea (A080588).

\section{References}

1 McDonald HR, Verre WP, Aaberg TM. Surgical management of idiopathic epiretinal membranes. Ophthalmology 1986; 93: 978-983.

2 Stern WH, Fisher SK, Anderson DH, O'Donnell JJ, Erickson PA, Lewis GP et al. Epiretinal membrane formation after vitrectomy. Am J Ophthalmol 1982; 93: 757-772.

3 Kono T, Kohno T, Inomata H. Epiretinal membrane formation. Light and electron microscopic study in an experimental rabbit model. Arch Ophthalmol 1995; 113: 359-363.

4 Forte R, Pascotto F, de Crecchio G. Visualization of vitreomacular tractions with en face optical coherence tomography. Eye 2007; 21: 1391-1394.

5 Koizumi H, Spaide RF, Fisher YL, Freund KB, Klancnik Jr JM, Yannuzzi LA. Three-dimensional evaluation of vitreomacular traction and epiretinal membrane using spectral-domain optical coherence tomography. Am J Ophthalmol 2008; 145: 509-517.

6 Suzuki T, Terasaki H, Niwa T, Mori M, Kondo M, Miyake Y. Optical coherence tomography and focal macular electroretinogram in eyes with epiretinal membrane and macular pseudohole. Am J Ophthalmol 2003; 136: 62-67.

7 Davison JA, Chylack LT. Clinical application of the lens opacities classification system III in the performance of phacoemulsification. J Cataract Refract Surg 2003; 29: 138-145.

8 Kwok AK, Lai TY, Yew DT, Li WW. Internal limiting membrane staining with various concentrations of indocyanine green dye under air in macular surgeries. Am J Ophthalmol 2003; 136: 223-230.

9 Matsumoto C, Arimura E, Okuyama S, Takada S, Hashimoto S, Shimomura Y. Quantification of metamorphopsia in patients with epiretinal membranes. Invest Ophthalmol Vis Sci 2003; 44: 4012-4016.

10 Gupta P, Sadun AA, Sebag J. Multifocal retinal contraction in macular pucker analyzed by combined optical coherence tomography/scanning laser ophthalmoscopy. Retina 2008; 28: $447-452$.

11 Grewing R, Mester U. Results of surgery for epiretinal membranes and their recurrences. Br J Ophthalmol 1996; 80: 323-326.

12 Rohrschneider K. Determination of the location of the fovea on the fundus. Invest Ophthalmol Vis Sci 2004; 45: 3257-3258.

13 Minchiotti S, Stampachiacchiere B, Micera A, Lambiase A, Ripandelli G, Billi B et al. Human idiopathic epiretinal membranes express NGF and NGF receptors. Retina 2008; 28: 628-637.

14 Niwa T, Terasaki H, Kondo M, Piao CH, Suzuki T, Miyake Y. Function and morphology of macula before and after removal of idiopathic epiretinal membrane. Invest Ophthalmol Vis Sci 2003; 44: 1652-1656.

15 Massin P, Allouch C, Haouchine B, Metge F, Paques M, Tangui L et al. Optical coherence tomography of idiopathic macular epiretinal membranes before and after surgery. Am J Ophthalmol 2000; 130: 732-739.

16 Wilkins JR, Puliafito CA, Hee MR, Duker JS, Reichel E, Coker JG et al. Characterization of epiretinal membranes 
using optical coherence tomography. Ophthalmology 1996; 103: 2142-2151.

17 Suh MH, Seo JM, Park KH, Yu HG. Associations between macular findings by optical coherence tomography and visual outcomes after epiretinal membrane removal. Am J Ophthalmol 2009; 147: 473-480.e3.

18 Asaria R, Garnham L, Gregor ZJ, Sloper JJ. A prospective study of binocular visual function before and after successful surgery to remove a unilateral epiretinal membrane. Ophthalmology 2008; 115: 1930-1937.

19 Villate N, Lee JE, Venkatraman A, Smiddy WE. Photoreceptor layer features in eyes with closed macular holes: optical coherence tomography findings and correlation with visual outcomes. Am J Ophthalmol 2005; 139: 280-289.

20 Lardenoye CW, Probst K, DeLint PJ, Rothova A. Photoreceptor function in eyes with macular edema. Invest Ophthalmol Vis Sci 2000; 41: 4048-4053.
21 Murakami T, Tsujikawa A, Ohta M, Miyamoto K, Kita M, Watanabe $\mathrm{D}$ et al. Photoreceptor status after resolved macular edema in branch retinal vein occlusion treated with tissue plasminogen activator. Am J Ophthalmol 2007; 143: 171-173.

22 Richter-Mueksch S, Vecsei-Marlovits PV, Sacu SG, Kiss CG, Weingessel B, Schmidt-Erfurth U. Functional macular mapping in patients with vitreomacular pathologic features before and after surgery. Am J Ophthalmol 2007; 144: 23-31.

23 Donati G, Kapetanios AD, Pournaras CJ. Complications of surgery for epiretinal membranes. Graefes Arch Clin Exp Ophthalmol 1998; 236: 739-746.

24 Wong JG, Sachdev N, Beaumont PE, Chang AA. Visual outcomes following vitrectomy and peeling of epiretinal membrane. Clin Experiment Ophthalmol 2005; 33: 373-378.

25 Thompson JT. Epiretinal membrane removal in eyes with good visual acuities. Retina 2005; 25: 875-882. 